\title{
A DISTRIBUTION LAW FOR CCS AND A NEW CONGRUENCE RESULT FOR THE $\pi$-CALCULUS *
}

\author{
DANIEL HIRSCHKOFF AND DAMIEN POUS \\ e-mail address: \{Daniel.Hirschkoff,Damien.Pous\}@ens-lyon.fr \\ ENS Lyon, Université de Lyon, LIP (UMR 5668 CNRS ENS Lyon UCBL INRIA), France
}

\begin{abstract}
We give an axiomatisation of strong bisimilarity on a small fragment of CCS that does not feature the sum operator. This axiomatisation is then used to derive congruence of strong bisimilarity in the finite $\pi$-calculus in absence of sum. To our knowledge, this is the only nontrivial subcalculus of the $\pi$-calculus that includes the full output prefix and for which strong bisimilarity is a congruence.
\end{abstract}

\section{INTRODUCTION}

In this paper, we study strong bisimilarity on two process calculi. More precisely, we establish an axiomatisation for strong bisimilarity on a very restricted fragment of CCS, and then use this axiomatisation to derive a new congruence result for the $\pi$-calculus.

We first focus on microCCS ( $\mu \mathrm{CCS}$ ), the subcalculus of CCS that only features prefix and parallel composition. Our main result on $\mu \mathrm{CCS}$ is that adding the following distribution law

$$
\eta \cdot(P|\eta \cdot P| \ldots \mid \eta \cdot P)=\eta \cdot P|\eta \cdot P| \ldots \mid \eta \cdot P
$$

to the laws of an abelian monoid for parallel composition yields a complete axiomatisation of strong bisimilarity (in the law above, $\eta$ is a CCS prefix, of the form $a$ or $\bar{a}$, and $P$ is any CCS process - the same number of copies of $P$ appear on both sides of the equation).

The distribution law is not new: it is mentioned - among other 'mixed equations' relating prefixed terms and parallel compositions - in a study of bisimilarity on normed PA processes [10. In our setting, this equality can be oriented from left to right to rewrite processes into normal forms, which intuitively exhibit as much concurrency as possible. Strong bisimilarity $(\sim)$ between processes is then equivalent to equality of their normal forms. This rewriting phase allows us to actually compute unique decompositions of processes into prime processes, in the sense of [12]: a process $P$ is prime if $P$ is not bisimilar to the inactive process $\mathbf{0}$ and if $P \sim Q \mid R$ implies $Q \sim \mathbf{0}$ or $R \sim \mathbf{0}$.

The distribution law is an equational schema, corresponding to an infinite family of axioms, of the form $\eta \cdot\left(P \mid(\eta \cdot P)^{k}\right)=(\eta \cdot P)^{k+1}$, for $k \geq 1$ (where $Q^{k}$ denotes the $k$-fold

1998 ACM Subject Classification: F.e.2.

Key words and phrases: Bisimulation, process algebra, CCS, $\pi$-calculus, axiomatisation.

* A preliminary version of this work appeared in 9 .

\section{LOGICAL METHODS} IN COMPUTER SCIENCE
DOI:10.2168/LMCS-4 (2:4) 2008
Daniel Hirschkoff and Damien Pous Creative Commons 
parallel composition of process $Q$ ). Some of these axioms are related. For instance, we can derive the 6-ary instance of the distribution law (corresponding to $k=5$ ) using the binary and the ternary instances: first rewrite $(\eta \cdot P)^{6}$ three times using the binary instance, yielding $(\eta \cdot(P \mid \eta \cdot P))^{3}$; then use the ternary instance to rewrite the latter process into $\eta \cdot((P \mid \eta . P)|\eta \cdot(P \mid \eta . P)| \eta \cdot(P \mid \eta . P))$; finally, use the binary instance twice to get $\eta .\left(P \mid(\eta \cdot P)^{5}\right)$. On the other hand, instances of the distribution law where the prefixed term occurs a prime number of times on the right hand side cannot be derived using other instances. We formalise this argument to show that there exists no finite axiomatisation of $\sim$ on $\mu$ CCS in Sect. 3 ,

We are also interested in this paper in the $\pi$-calculus, and, more precisely, in congruence properties of strong bisimilarity in this formalism. Because of the presence of the input prefix, and of the related phenomenon of name-passing, bisimilarity is more complex in the $\pi$-calculus than in CCS. In particular, both early and late bisimilarity [15, that differ in their treatment of name substitution, fail to be congruences in the full $\pi$-calculus.

There exist subcalculi of the $\pi$-calculus for which strong bisimilarity is a congruence (we discuss these in Sect. 6). When this is the case, this equivalence coincides with ground bisimilarity $\left(\sim_{\mathrm{g}}\right)$, which allows one to consider a single fresh name when analysing an input transition, instead of the usual quantification involving all free names of the process. Congruence of strong bisimilarity is hence an important property: not only is it necessary in order to reason in a compositional way, but it also helps making bisimulation proofs simpler, by reducing the number of cases to analyse.

In the full $\pi$-calculus, in order to get congruence, one has to work with Sangiorgi's open bisimilarity [14, which has a more involved definition than the early and late variants. Tools like the Mobility Workbench [16], for instance, have adopted this equivalence on processes.

Technically, the key property which is necessary in order to derive congruence of $\sim_{\mathrm{g}}$ in the $\pi$-calculus is substitution closure: we say that a relation $\mathcal{R}$ between processes is closed under substitution if whenever $P \mathcal{R} Q$, then $P \sigma \mathcal{R} Q \sigma$ for any substitution $\sigma$ mapping names to names. In calculi like CCS or the $\pi$-calculus, where interaction arises from the synchronisation between an emitter and a receiver, substitution closure is a demanding property. Indeed, applying a substitution may have the effect of identifying two names, thus triggering new possibilities of interaction.

Before addressing substitution closure for $\sim_{\mathrm{g}}$ in the $\pi$-calculus, we analyse this property in the simpler setting of (subsets of) CCS in Sect. 4. We show in particular that strong bisimilarity is closed under substitution in $\mu \mathrm{CCS}$, but that it is not as soon as we add the choice operator, although being a congruence.

At the heart of our proof of congruence in the $\pi$-calculus is a notion that we call mutual desynchronisation, and that corresponds to the existence of processes $P, P_{12}, P_{21}$ such that $P \stackrel{\eta_{1}}{\longrightarrow} \stackrel{\eta_{2}}{\longrightarrow} P_{12}$ and $P \stackrel{\eta_{2}}{\longrightarrow} \stackrel{\eta_{1}}{\longrightarrow} P_{21}$, for two distinct actions $\eta_{1}$ and $\eta_{2}$, and with $P_{12}$ behaviourally equivalent to $P_{21}$. (We do not specify the shape of actions, nor the behavioural equivalence we refer to, because we shall be reasoning about mutual desynchronisations both in $\mu \mathrm{CCS}$ and in the $\pi$-calculus.) We additionally require in the two sequences of transitions from $P$ to $P_{12}$ and $P_{21}$ respectively that the second prefix being fired should occur under the first prefix in $P$.

We discuss the relationship between substitution closure and mutual desynchronisations in Sect. 4.1, and show that the latter do not arise in $\mu$ CCS (which is a way to prove that $\sim$ is closed under substitution in this calculus). This is essentially due to the fact that our 
axiomatisation of $\sim$ on $\mu \mathrm{CCS}$ does not allow one to match the firing of two distinct prefixes that are concurrent using two prefixes that occur in sequence in a process.

In relation with the latter observation, we then argue in Sect. 4.2 that noninterleaving semantics, for which concurrency cannot be reduced to nondeterminism, are more likely to be substitution closed: we prove that this is the case for Castellani and Hennessy's distributed bisimilarity [5] in $\mu \mathrm{CCS}$ extended with choice.

Coming back to the $\pi$-calculus, we exploit a transfer property that allows us to derive from the absence of mutual desynchronisations in $\mu$ CCS the same result in $\pi_{0}$, the finite, sum-free $\pi$-calculus. This entails that ground, early, late and open bisimilarities coincide on $\pi_{0}$, and are congruences. It is known [15, that bisimilarity in the $\pi$-calculus fails to be a congruence as soon as we have prefix, parallel composition, restriction and replication. The problem of congruence of $\sim_{\mathrm{g}}$ on $\pi_{0}$ is mentioned as an open question in [15. Chapter 5], and is known since at least 1998 [2]. To our knowledge, this is the first congruence result for a subcalculus of the $\pi$-calculus that includes the full output prefix (see Sect. 66 for a discussion on this).

Paper outline. We introduce $\mu \mathrm{CCS}$ and the distribution law in Sect. 1. Section 2 is devoted to the characterisation of $\sim$ on $\mu$ CCS using normal forms. In Sect. 3, we prove that no finite axiomatisation of $\sim$ on $\mu$ CCS exists. We discuss the substitution closure property, and establish it for distributed bisimilarity in an extension of $\mu$ CCS, in Sect. 4 . Section 5 presents the proof of our congruence result in the $\pi$-calculus, and we give concluding remarks in Sect. 6.

This paper is an extended version of [9]. In particular, we provide more detailed proofs in Sect. 3, the material in Sect. 4.2, that discusses substitution closure and noninterleaving semantics, is new.

\section{MicroCCS Processes and Normal Forms}

We consider an infinite set $\mathcal{N}$ of names, and let $a, b \ldots$ range over names. We define on top of $\mathcal{N}$ the set of processes of $\mu \mathrm{CCS}$, the finite, public (that is, without restriction), sum-free CCS calculus, as follows, where $P, Q, R \ldots$ range over processes:

$$
\eta::=a|\bar{a}, \quad P::=\mathbf{0}| \eta \cdot P|P| Q
$$

$\mathbf{0}$ is the nil process. $\eta$ ranges over interactions (also called visible actions), and we let $\bar{\eta}$ stand for the coaction associated to $\eta$ (we let $\overline{\bar{\eta}}=\eta$ ). For $k>0$, we write $P^{k}$ for the parallel composition of $k$ copies of $P$, and we write $\prod_{i \in I} P_{i}$ for the parallel composition of all processes $P_{i}$ for $i \in I$.

Structural congruence, written $\equiv$, is defined as the smallest congruence satisfying the following laws:

$$
\left(C_{1}\right) \quad P|Q \equiv Q| P \quad\left(C_{2}\right) \quad P|(Q \mid R) \equiv(P \mid Q)| R \quad\left(C_{3}\right) \quad P \mid \mathbf{0} \equiv P
$$

We introduce a labelled transition system (LTS) for $\mu$ CCS. Actions labelling transitions are either interactions, or a special silent action, written $\tau$. We use $\mu$ to range over actions. It can be noted that the syntax of $\mu$ CCS does not include a construction of the form $\tau . P-$ see Remark 2.8 below. 
Definition 1.1 (Operational semantics and behavioural equivalence). The LTS for $\mu$ CCS is given by the following rules:

$$
\eta . P \stackrel{\eta}{\rightarrow} P \quad \frac{P \stackrel{\eta}{\rightarrow} P^{\prime} \quad Q \stackrel{\bar{\eta}}{\rightarrow} Q^{\prime}}{P\left|Q \stackrel{\tau}{\rightarrow} P^{\prime}\right| Q^{\prime}} \quad \frac{P \stackrel{\mu}{\rightarrow} P^{\prime}}{P\left|Q \stackrel{\mu}{\rightarrow} P^{\prime}\right| Q} \quad \frac{P \stackrel{\mu}{\rightarrow} P^{\prime}}{Q|P \stackrel{\mu}{\rightarrow} Q| P^{\prime}}
$$

A bisimulation is a symmetrical relation $\mathcal{R}$ between processes such that whenever $P \mathcal{R} Q$ and $P \stackrel{\mu}{\rightarrow} P^{\prime}$, there exists $Q^{\prime}$ such that $Q \stackrel{\mu}{\rightarrow} Q^{\prime}$ and $P^{\prime} \mathcal{R} Q^{\prime}$.

Bisimilarity, written $\sim$, is the union of all bisimulations.

Definition 1.2 (Size). Given $P, \#(P)$ (called the size of $P$ ) is defined by:

$$
\#(\mathbf{0}) \stackrel{\text { def }}{=} 0 \quad \#\left(P_{1} \mid P_{2}\right) \stackrel{\text { def }}{=} \#\left(P_{1}\right)+\#\left(P_{2}\right) \quad \#(\eta \cdot P) \stackrel{\text { def }}{=} 1+\#(P) .
$$

Lemma 1.3. $P \equiv Q$ implies $P \sim Q$ which in turn implies $\#(P)=\#(Q)$.

Proof. The first implication follows by showing that the laws of $\equiv$ are sound for $\sim$, and that $\sim$ is preserved by parallel composition and prefix.

Assume then by contradiction that there exist $P, Q$ such that $P \sim Q$ and $\#(P)<\#(Q)$; and choose such $P$ with minimal size. $Q$ has at least one prefix: $Q \stackrel{\eta}{\rightarrow} Q^{\prime}$ and we get $P \stackrel{\eta}{\rightarrow} P^{\prime}$ with $P^{\prime} \sim Q^{\prime}$. We deduce that $\#\left(P^{\prime}\right)<\#(P)$ and $\#\left(P^{\prime}\right)<\#\left(Q^{\prime}\right)$, which contradicts the minimality hypothesis.

Definition 1.4 (Distribution law). The distribution law is given by the following equation, where the same number of copies of $P$ appears on both sides:

$$
\eta \cdot(P|\eta \cdot P| \ldots \mid \eta \cdot P)=\eta \cdot P|\eta \cdot P| \ldots \mid \eta \cdot P .
$$

We shall use this equality, oriented from left to right, to rewrite processes. We write $P \rightsquigarrow P^{\prime}$ when there exist $P_{1}, P_{2}$ such that $P \equiv P_{1}, P_{2} \equiv P^{\prime}$ and $P_{2}$ is obtained from $P_{1}$ by replacing a sub-term of the form of the left-hand side process with the right-hand side process.

Remark 1.5 (On the distribution law and PA). Among the studies about properties of $\sim$ in process algebras that include parallel composition (see [1] for a recent survey on axiomatisations), some works focus on calculi where parallel composition is treated as a primitive operator (as opposed to being expressible using sum or other constructs like the left merge operator). As mentioned above, particularly relevant to this work is [10], where Hirshfeld and Jerrum "develop a structure theory for PA that completely classifies the situations in which a sequential composition of two processes can be bisimilar to a parallel composition". [10] establishes decidability of $\sim$ for normed PA processes: in that setting, the formal analogue of the distribution law (Def. 1.4) holds with $\eta$ and $P$ being two processes - the 'dot' operator is a general form of sequential composition. This equality is valid in [10] whenever $\eta$ is a 'monomorphic process', meaning that $\eta$ can only reduce to $\mathbf{0}$ (which corresponds to $\mu \mathrm{CCS}$ ), or to $\eta$ itself. [7] presents a finite axiomatisation of PA that exploits the operators of sum and left merge.

Lemma 1.6. The relation $\rightsquigarrow$ is strongly normalising and confluent.

Proof. If $P \rightsquigarrow P^{\prime}$ then the weight of $P^{\prime}$ (defined as sum of the depths of all prefixes occurring in $P^{\prime}$ ) is strictly smaller than the weight of $P$, whence the strong normalisation. We then remark that $\rightsquigarrow$ is locally confluent, and conclude with Newman's Lemma. 
Thus, for any process $P, \rightsquigarrow$ defines a normal form unique up to $\equiv$, that will be denoted by $\mathrm{n}(P)$. We let $A, B, \ldots$ range over normal forms.

The following lemma states that $\rightsquigarrow$ preserves bisimilarity:

Lemma 1.7. If $P \rightsquigarrow P^{\prime}$, then $P \sim P^{\prime}$. For any $P, P \sim \mathrm{n}(P)$.

Proof. The relation $\left(\rightsquigarrow \cup(\rightsquigarrow)^{-1} \cup \equiv\right)$ is a bisimulation.

\section{Characterisation of Bisimilarity in MicroCCS}

Our characterisation of $\sim$ on $\mu \mathrm{CCS}$ makes use of the notion of decomposition into prime processes, defined as follows:

Definition 2.1. A process $P$ is prime if $P \not \mathbf{0}$ and $P \sim P_{1} \mid P_{2}$ implies $P_{1} \sim \mathbf{0}$ or $P_{2} \sim \mathbf{0}$.

When $P \sim P_{1}|\ldots| P_{n}$ where the $P_{i}$ s are prime, we shall call $P_{1}|\ldots| P_{n}$ a prime decomposition of $P$.

Proposition 2.2 (Unique decomposition). Any process admits a prime decomposition which is unique up to bisimilarity: if $P_{1}|\ldots| P_{n}$ and $Q_{1}|\ldots| Q_{m}$ are two prime decompositions of the same process, then $n=m$ and there exists a permutation $f$ of $[1 . . n]$ such that $P_{i} \sim Q_{f(i)}$ for all $i \in[1 . . n]$.

Proof. Similar to the proof of [13, Theorem 4.3.1]: the case of $\mu$ CCS is not explicitly treated in that work, but the proof can be adapted rather easily.

An immediate consequence of the above result is the following property:

Corollary 2.3 (Cancellation). For all $P, Q, R, P|R \sim Q| R$ implies $P \sim Q$.

Note that this is not true in presence of replication: $a|! a \sim 0| ! a$, but $a \nsim \mathbf{0}$.

The characterisation of $\sim$ using the distribution law follows from the observation that if a normal form is a prefixed process, then it is prime. This idea is used in the proof of Lemma 2.5. We first establish a technical result, that essentially exploits the same argument as the proof of Theorem 4.2 in [10].

Lemma 2.4. If $\eta . P \sim Q \mid Q^{\prime}$, with $Q, Q^{\prime} \nsim \mathbf{0}$, then there exist $A$ and $k>1$ such that $\eta . P \sim(\eta \cdot A)^{k}$ and $\eta . A$ is a normal form.

Proof. By Lemma 1.7, we have $\eta . P \sim \mathrm{n}\left(Q \mid Q^{\prime}\right)$. Furthermore, we have that $\mathrm{n}\left(Q \mid Q^{\prime}\right) \equiv$ $\prod_{i \leq k} \eta_{i} . A_{i}$, where $k>1$ and the processes $\eta_{i} . A_{i}$ are in normal form.

Since the $\eta$ prefix must be triggered to answer any challenge from the right hand side, we have $\eta_{i}=\eta$ and $P \sim A_{i} \mid \prod_{l \neq i} \eta$. $A_{l}$ for all $i \leq k$. In particular, when $i \neq j$, we have $P \sim A_{i}\left|\eta \cdot A_{j}\right| \prod_{l \notin\{i, j\}} \eta \cdot A_{l} \sim \eta \cdot A_{i}\left|A_{j}\right| \prod_{l \notin\{i, j\}} \eta \cdot A_{l}$ and hence, by Corollary 2.3, $A_{i}\left|\eta \cdot A_{j} \sim \eta \cdot A_{i}\right| A_{j}$. By reasoning on the sizes of the parallel components in the prime decompositions of these two terms, we conclude that $\eta . A_{i} \sim \eta$. $A_{j}$ for all $i, j \leq k$.

Hence, we have $\eta . P \sim\left(\eta . A_{1}\right)^{k}$ with $k>1$ and $\eta . A_{1}$ is a normal form. 
Lemma 2.5. Let $A, B$ be two normal forms, $A \sim B$ implies $A \equiv B$.

Proof. We show by induction on $n$ that for all $A$ with $\#(A)=n$, we have

(i) if $A$ is a prefixed process, then $A$ is prime;

(ii) for any $B, A \sim B$ implies $A \equiv B$.

The case $n=0$ is immediate. Assume that the property holds for all $i<n$, with $n \geq 1$.

(i) We write $A=\eta \cdot A^{\prime}$, and assume by contradiction $A \sim P_{1} \mid P_{2}$ with $P_{1}, P_{2} \nsim \mathbf{0}$. By Lemma 2.4, we have $A \sim(\eta \cdot B)^{k}$ with $k>1$ and $\eta . B$ in normal form. By triggering the prefix on the left hand side, we have $A^{\prime} \sim B \mid(\eta \cdot B)^{k-1}$. It follows by induction that $A^{\prime} \equiv B \mid(\eta \cdot B)^{k-1}$ (using property $\left.(i i)\right)$, and hence $A \equiv \eta \cdot\left(B \mid(\eta \cdot B)^{k-1}\right.$, which is in contradiction with the fact that $A$ is in normal form.

(ii) Assume now $A \sim B$.

- If $A$ is a prefixed process, $B$ is prime by the previous point $(\#(B)=\#(A)$ by Lemma 1.3). Necessarily, $A \equiv \eta \cdot A^{\prime}$ and $B \equiv \eta \cdot B^{\prime}$ with $A^{\prime} \sim B^{\prime}$. By induction, this entails $A^{\prime} \equiv B^{\prime}$, and $A \equiv B$.

- Otherwise, $A=\eta_{1} \cdot A_{1}|\ldots| \eta_{k} . A_{k}$ with $k>1$, and we know by induction (property (i)) that $\eta_{i} . A_{i}$ is prime for all $i \leq k$. Similarly, we have $B=\eta_{1}^{\prime} . B_{1}|\ldots| \eta_{l}^{\prime} . B_{l}$ with $\eta_{i}^{\prime} \cdot B_{i}$ prime for all $i \leq l$.

By Proposition $2.2, k=m$ and $\eta_{i} . A_{i} \sim \eta_{i}^{\prime}$. $B_{i}$ (up to a permutation of the indices), which gives $\eta_{i}^{\prime}=\eta_{i}$ and $A_{i} \sim B_{i}$ for all $i \leq k$. By induction, we deduce $A_{i} \equiv B_{i}$ for all $i$, which finally implies $A \equiv B$.

Lemmas 1.7 and 2.5 allow us to deduce the following result.

Theorem 2.6. Let $P, Q$ be two $\mu C C S$ processes. Then $P \sim Q$ iff $\mathrm{n}(P) \equiv \mathrm{n}(Q)$.

Remark 2.7 (Unique decomposition of processes). Our proof relies on unique decomposition of processes (Prop. 2.2), that first appeared in [12]. Unique decomposition has been established for a variety of process algebras, and used as a way to prove decidability of behavioural equivalence and to give complexity bounds for the associated decision procedure ([11, 3] cite relevant references).

In the present study, beyond the existence of a unique decomposition, we are interested in a syntactic characterisation of $\sim$ (which will in particular allow us to derive Lemma 4.4 below). In this sense, our work is close to [6], where the notion of maximally parallel process in CCS (with choice) is studied. [6] defines a rewriting process through which maximally parallel normal forms can be computed, and shows that in the case of $\mu \mathrm{CCS}$, such normal forms are unique. However, no syntactical characterisation of the set of normal forms is presented, and such a characterisation cannot be directly deduced from the (rather involved) definition of the rewriting process for full CCS.

We instead restrict ourselves to $\mu$ CCS from the start, and rely explicitly on the distribution law in order to 'extract' prime components of processes.

Remark 2.8 ( $\tau$ prefix and weak bisimilarity). We do not address weak bisimilarity in the present work. In $\mu \mathrm{CCS}$, strong and weak bisimilarity coincide, i.e., the internal transitions of processes are completely determined by the visible actions (interactions). This is essentially due to the absence of restriction in the calculus. When including $\tau$ prefixes in the syntax, it can be proved that adding the law $\tau . P=P$ is enough to characterise weak bisimilarity. The $\tau$ prefix is usually absent in the $\pi$-calculus, to which we shall move in Sect. 5. Since some results on CCS will be transferred to the $\pi$-calculus, we did not include this construct in $\mu \mathrm{CCS}$. 


\section{Nonexistence of a Finite Axiomatisation}

We let $M, N$ range over $\mu$ CCS terms with variables (this corresponds to the grammar $M::=\mathbf{0}|\eta \cdot M| M|M| X$, and we use $X, Y \ldots$ to range over term variables). A ground term is a term with no occurrence of variables. Instantiations are mappings from variables to terms, and their domain are naturally extended to terms. We use $\rho$ to range over instantiations. Applying $\rho$ to $M$ yields a term written $M \rho . \rho$ is a ground instantiation if for all terms $M, M \rho$ is a ground term. Any two terms $M, N$ define an equation, written $M=N$.

Definition 3.1 (Axiomatic equality). Given a set $\mathcal{E}$ of equations, we shall write $\mathcal{E} \vdash M=N$ whenever $M=N$ can be derived in equational logic using equations from $\mathcal{E}$.

We let $\mathcal{D}$ stand for the set of equations consisting of the three axioms of structural congruence $\left(C_{1}, C_{2}, C_{3}\right)$, and all the distribution axioms $\left(\left(D_{i}\right)_{i \geq 1}\right)$ :

$$
\left(D_{i}\right): \eta \cdot\left(P \mid(\eta \cdot P)^{i}\right)=(\eta \cdot P)^{i+1}, i \geq 1 .
$$

$\mathcal{D}_{k}$ stands for the finite restriction of $\mathcal{D}$ where only the first $k$ distribution axioms are included $\left(\left(D_{i}\right)_{1 \leq i \leq k}\right)$.

Equations of $\mathcal{D}$ are obviously sound for $\sim$. Ground completeness is given by the following proposition, which holds by Theorem 2.6.

Proposition 3.2 (Completeness). For any processes $P, Q$,

$$
P \sim Q \quad \text { iff } \quad \mathcal{D} \vdash P=Q
$$

We now analyse the distribution law using a rather classical approach [1]. We show that $\mathcal{D}$ is $\omega$-complete, that is, complete w.r.t. the extensional equality derived from strong bisimilarity. Since, by Lemma 3.8 below, $\mathcal{D}$ is intrinsically infinite, we derive impossibility of a finite axiomatisation of $\sim$ on $\mu \mathrm{CCS}$, by using compactness arguments.

Definition 3.3 (Extensional equality). Two terms $M$ and $N$ are extensionally equal, written $M \sim_{\omega} N$, whenever for any ground instantiation $\rho$, it holds that $M \rho \sim N \rho$. An equation $M=N$ is said to be correct if $M \sim_{\omega} N$.

Our proof of $\omega$-completeness essentially relies on the methodology developped in [8]; the idea is to replace variables by small terms that can easily be distinguished.

Lemma 3.4. Let $M$ be a term whose variables all belong to $\left\{X_{i}\right\}_{i \in I}$, and let $\left\{a_{i}\right\}_{i \in I}$ be a collection of distinct names that do not occur in $M$.

$$
\mathrm{n}\left(M\left\{a_{i} .0 / X_{i}\right\}\right) \equiv \mathrm{n}(M)\left\{a_{i} .0 / X_{i}\right\}
$$

Proof. We proceed by well founded induction over the termination of $\rightsquigarrow$.

- If $M$ is in normal form, we just have to check that $M\left\{a_{i} .0 / X_{i}\right\}$ is in normal form. This is true because the $a_{i}$ are distinct and do not appear in $M$.

- Otherwise, if $M \rightsquigarrow N$, we check that $M\left\{a_{i} .0 / X_{i}\right\} \rightsquigarrow N\left\{a_{i} .0 / X_{i}\right\}$ so that:

$$
\begin{array}{rlr}
\mathrm{n}\left(M\left\{a_{i} .0 / X_{i}\right\}\right) & \equiv \mathrm{n}\left(N\left\{a_{i} .0 / X_{i}\right\}\right) & \text { (by confluence) } \\
& \equiv \mathrm{n}(N)\left\{a_{i} .0 / X_{i}\right\} & \text { (by induction) } \\
& \equiv \mathrm{n}(M)\left\{a_{i} .0 / X_{i}\right\} & \text { (by confluence) }
\end{array}
$$


Lemma 3.5. Let $M, N$ be two terms whose variables all belong to $\left\{X_{i}\right\}_{i \in I}$, and let $\left\{a_{i}\right\}_{i \in I}$ be a collection of distinct names that do not occur in $M$ nor in $N$.

- If $\mathcal{D} \vdash M=N$ then $\mathcal{D} \vdash M \rho=N \rho$ for any instantiation $\rho$;

- if $M\left\{a_{i} .0 / X_{i}\right\} \sim N\left\{a_{i} .0 / X_{i}\right\}$ then $\mathcal{D} \vdash M=N$.

Proof. The first point is standard, and proved by induction over the derivation tree.

For the second property, we know by Theorem 2.6 that $\mathrm{n}\left(M\left\{a_{i} .0 / X_{i}\right\}\right) \equiv \mathrm{n}\left(N\left\{a_{i} .0 / X_{i}\right\}\right)$. By Lemma 3.4, we can deduce $\mathrm{n}\left(M\left\{a_{i} .0 / X_{i}\right\}\right) \equiv \mathrm{n}(M)\left\{a_{i} .0 / X_{i}\right\}$, and $\mathrm{n}\left(N\left\{a_{i} .0 / X_{i}\right\}\right) \equiv$ $\mathrm{n}(N)\left\{a_{i} .0 / X_{i}\right\}$. Hence we have $\mathrm{n}(M) \equiv \mathrm{n}(N)$, and $\mathcal{D} \vdash M=N$ holds.

Theorem 3.6 ( $\omega$-completeness). For any terms $M, N$,

$$
M \sim_{\omega} N \quad \text { iff } \mathcal{D} \vdash M=N .
$$

Proof. Using Lemma 3.5, $\omega$-completeness boils down to the completeness of $\mathcal{D}$ for ground terms (Prop. 3.2).

Notice that the proof of Theorem 3.6 relies on the existence of an infinite number of names. The following result is standard.

Lemma 3.7 (Compactness). For any terms $M, N$,

$$
\mathcal{D} \vdash M=N \quad \text { iff } \quad \mathcal{D}_{k} \vdash M=N \text { for some } k .
$$

Proof. Equational proofs are finite objects.

Lemma 3.8. Let a be a name, for any number $k$, there exists $n$ such that:

$$
\mathcal{D}_{k} \forall a \cdot a^{n}=a^{n+1} .
$$

Remember that $a^{n}$ stands for the $n$-ary parallel composition of $a . \mathbf{0}$, so that this equality is an instance of axiom $\left(D_{n}\right)$.

Proof. Let $n$ be a number strictly greater than $k$ such that $n+1$ is prime, and let $\theta(P, Q)$ denote the predicate: " $P \sim Q \sim a^{n+1}, P \equiv a . P^{\prime}$, and $Q \equiv Q_{1} \mid Q_{2}$ with $Q_{1}, Q_{2} \not \equiv \mathbf{0}$ ".

Assume $\mathcal{D}_{k} \vdash a . a^{n}=a^{n+1}$, and consider the shortest proof of $\mathcal{D}_{k} \vdash P=Q$ for some processes $P, Q$ such that either $\theta(P, Q)$ or $\theta(Q, P)$. Since $\theta\left(a \cdot a^{n}, a^{n+1}\right)$ holds, such a minimal proof does exist. We reason about the last rule used in the derivation of this proof in equational logic. For syntactic reasons, this cannot be reflexivity, a contextual rule, nor one of the structural congruence axioms. It can be neither symmetry nor transitivity, since otherwise this would give a shorter proof satisfying $\theta$. The only possibility is thus the use of one of the distribution axioms, say $D_{i}$ with $1 \leq i \leq k$ and $a^{n+1} \sim Q \equiv\left(a \cdot Q^{\prime}\right)^{i+1}$. By Lemma 1.3. since \# $\left(a^{n+1}\right)=n+1, i+1$ has to divide $n+1$. This is contradictory, because we have $2 \leq i+1 \leq k+1<n+1$, and $n+1$ is prime.

We can finally prove the nonexistence of a finite axiomatisation of $\sim$ on $\mu \mathrm{CCS}$. The proof corresponds to a standard application of the Compactness Theorem [1].

Theorem 3.9 (No finite axiomatisation of $\sim$ ). For any finite set of correct equations $\mathcal{E}$, there exist processes $P$ and $Q$ such that $P \sim Q$ but $\mathcal{E} \forall P=Q$.

Proof. By correctness, for any equation $M=N$ in $\mathcal{E}, M \sim_{\omega} N$. Hence, by $\omega$-completeness we can prove any equation of $\mathcal{E}$ using $\mathcal{D}$. By Lemma 3.7, and since $\mathcal{E}$ is finite, there exists $k$ such that $\mathcal{D}_{k} \vdash \mathcal{E}$. By Lemma 3.8, there exists $n$ such that $a . a^{n} \sim a^{n+1}$ and $\mathcal{D}_{k} \nvdash a \cdot a^{n}=a^{n+1}$; and thus, $\mathcal{E} \nvdash a \cdot a^{n}=a^{n+1}$. 


\section{On Substitution Closure of Bisimilarity}

We now discuss the property of substitution closure of behavioural equivalences in (subcalculi of) CCS. In the $\pi$-calculus, because of the input prefix, substitution closure is in general a necessary condition for bisimilarity to be a congruence. The notion of mutual desynchronisation, which we define in Sect. 4.1, allows us to show that $\sim$ is closed under substitution in $\mu \mathrm{CCS}$. This notion will be used to establish substitution closure (and then congruence) of $\sim_{\mathrm{g}}$ in $\pi_{0}$ in Sect. 5. We analyse substitution closure in an extension of $\mu \mathrm{CCS}$, both for strong bisimilarity and distributed bisimilarity, in Sect. 4.2 (the latter section is not technically necessary to establish the result on $\pi_{0}$, and can therefore be skipped).

4.1. Mutual Desynchronisations. In $\mu \mathrm{CCS}, \sim$ is closed under substitution. One way to prove that is to rely on the axiomatisation from Sect. 2, two processes related by an instance of the distribution law remain equivalent when a substitution mapping names to names is applied (we can show in particular that for any substitution $\sigma, \mathrm{n}(P \sigma) \equiv \mathrm{n}(\mathrm{n}(P) \sigma)$ ).

Here, we derive this result using an alternative general pattern, that corresponds to the proof of substitution closure of $\sim_{\mathrm{g}}$ in Sect. 5. To understand how the notion of mutual desynchronisation arises, we sketch the proof of substitution closure of $\sim$. Suppose for that $P \sim Q$, and consider a substitution $\sigma$. To prove $P \sigma \sim Q \sigma$, we reason by coinduction, and consider a transition $P \sigma \stackrel{\mu}{\rightarrow} P_{0}$. The difficult case arises when $\mu=\tau$, and the synchronisation follows from $P \stackrel{a}{\rightarrow} P_{1}, P \stackrel{\bar{b}}{\rightarrow} P_{2}$, with $\sigma(a)=\sigma(b)$. We observe that because we work in $\mu \mathrm{CCS}$, the transitions of $P$ to $P_{1}$ and $P_{2}$ are necessarily offered by distinct parallel components of $P$. $P$ can therefore do a transition along $a$ followed by a transition along $\bar{b}$ to some $P^{\prime}$, to which $Q$ can answer since $P \sim Q$. If $Q$ answers by firing two prefixes that belong to different parallel components ('concurrent prefixes'), we are done: we can infer a $\tau$ transition for $Q \sigma$, and conclude using coinduction. If this is not the case (i.e., if the $\bar{b}$ prefix fired by $Q$ was guarded by the $a$ prefix), we consider the sequence where $P$ performs the two transitions in the reversed order, first $\bar{b}$ then $a$, and reason similarly. Therefore, the only case where we cannot conclude occurs when $Q$ matches both sequences of transitions using causally dependent prefixes. This situation is depicted below; we will show that it cannot arise in $\mu \mathrm{CCS}$.

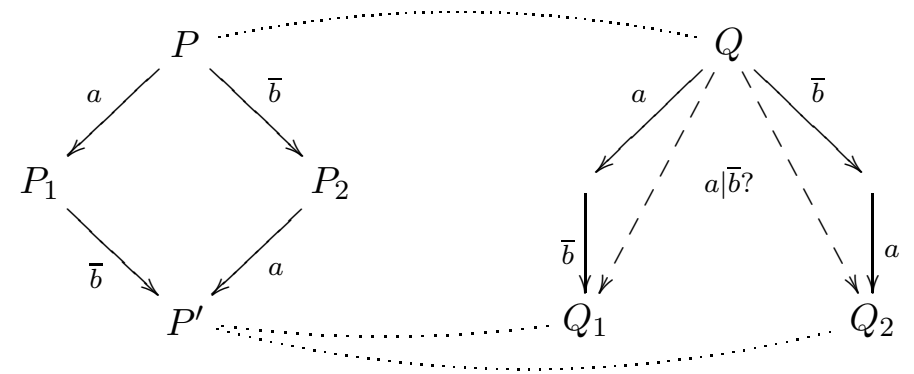

More precisely, we show that the situation on the right of this picture, where we notice that $Q_{1} \sim Q_{2}$ (both processes are bisimilar to $P^{\prime}$ ) cannot arise; we call such a - hypothetical - situation a mutual desynchronisation: 
Definition 4.1 (Mutual desynchronisation in $\mu \mathrm{CCS}$ ). We say that there exists a mutual desynchronisation in $\mu C C S$ whenever there are two prefixes $\eta_{1}, \eta_{2}$, and five $\mu$ CCS processes $S, S^{\prime}, T, T^{\prime}, R$ such that $\eta_{1} \neq \eta_{2}, S \stackrel{\eta_{1}}{\longrightarrow} S^{\prime}, T \stackrel{\eta_{2}}{\longrightarrow} T^{\prime}$ and $\eta_{2} . S\left|T^{\prime}\right| R \sim S^{\prime}\left|\eta_{1} . T\right| R$.

We recover the situation which is depicted above by taking $Q=\eta_{2} . S\left|\eta_{1} . T\right| R, \eta_{1}=a$, and $\eta_{2}=\bar{b}$. Such a notion is not specific to $\mu$ CCS: the proofs of Lemmas [5.6 and [5.7 will expose analogous situations in $\pi_{0}$.

Definition 4.2. We define, for any $\mu$ CCS process $P$ and prefix $\eta$, the contribution of $P$ at $\eta$, written $s_{\eta}(P)$, by

$$
\begin{array}{cl}
s_{\eta}(\mathbf{0}) \stackrel{\text { def }}{=} 0 & s_{\eta}\left(\eta^{\prime} \cdot P\right) \stackrel{\text { def }}{=} 0 \quad \text { if } \eta \neq \eta^{\prime} \\
s_{\eta}\left(P_{1} \mid P_{2}\right) \stackrel{\text { def }}{=} s_{\eta}\left(P_{1}\right)+s_{\eta}\left(P_{2}\right) & s_{\eta}(\eta \cdot P) \stackrel{\text { def }}{=} \#(\eta \cdot P)
\end{array}
$$

Intuitively, $s_{\eta}(P)$ is the total size of the parallel components of $P$ that start with the prefix $\eta$.

Lemma 4.3. $P \sim Q$ implies $s_{\eta}(P)=s_{\eta}(Q)$ for all $\eta$.

Proof. Follows from Theorem 2.6 and the observation that the distribution law preserves the contribution of a process at a given interaction prefix.

Lemma 4.4 (No mutual desynchronisation). There exists no mutual desynchronisation in $\mu C C S$.

Proof. Assume by contradiction that there are processes such that $P \stackrel{\eta_{1}}{\longrightarrow} P^{\prime}, Q \stackrel{\eta_{2}}{\longrightarrow} Q^{\prime}$ and $\eta_{2} . P\left|Q^{\prime}\right| R \sim P^{\prime}\left|\eta_{1} \cdot Q\right| R$.

By the cancellation property (Corollary 2.3), we have $\eta_{2} . P\left|Q^{\prime} \sim P^{\prime}\right| \eta_{1} \cdot Q$, hence for all $\eta, s_{\eta}\left(\eta_{2} . P \mid Q^{\prime}\right)=s_{\eta}\left(P^{\prime} \mid \eta_{1} \cdot Q\right)$ (Lemma 4.3).

Since $s_{\eta_{1}}\left(\eta_{2} . P \mid Q^{\prime}\right)=s_{\eta_{1}}\left(Q^{\prime}\right) \leq \#\left(Q^{\prime}\right)$ and $\left.s_{\eta_{1}}\left(P^{\prime} \mid \eta_{1} \cdot Q\right)\right) \geq s_{\eta_{1}}\left(\eta_{1} \cdot Q\right)=\#\left(Q^{\prime}\right)+2$, by taking $\eta=\eta_{1}$ we finally get $\#\left(Q^{\prime}\right) \geq \#\left(Q^{\prime}\right)+2$.

Lemma 4.4 will be used to show that a situation corresponding to a mutual desynchronisation cannot arise in $\pi_{0}$. Notice that the proof depends in an essential way on Lemma 4.3, which in turn relies on the axiomatisation of $\sim$ in $\mu \mathrm{CCS}$ (Theorem 2.6).

As a consequence of this result, we can deduce the following

Corollary 4.5 (Substitution closure of $\sim$ in $\mu \mathrm{CCS}$ ). In $\mu C C S, P \sim Q$ entails $P \sigma \sim Q \sigma$, for all substitution $\sigma$.

We now introduce an extension of $\mu \mathrm{CCS}$, called $\mu \mathrm{CCS}^{+}$, which is the calculus obtained by adding a sum operator over prefixed processes. The grammar of $\mu \mathrm{CCS}^{+}$is thus the following:

$$
S::=0|\eta \cdot P| S_{1}+S_{2}, \quad P::=S\left|P_{1}\right| P_{2} .
$$

If $I=[1 . . k]$, we write $\sum_{i \in I} S_{i}$ for $S_{1}+\cdots+S_{k}$. Like before, we use notation $\prod_{i} S_{i}$ for parallel compositions; when using this notation, we shall moreover implicitly assume that for all $i \in I, S_{i} \chi_{\mathrm{d}} \mathbf{0}$ (this is in particular the case in the statement of Lem. 4.11). We shall overload notations, and use $\sim$ to denote strong bisimilarity in $\mu \mathrm{CCS}^{+}$.

In $\mu \mathrm{CCS}^{+}, \sim$ is a congruence, but it is not closed under substitution. We have indeed

$$
a \mid \bar{b} \sim a \cdot \bar{b}+\bar{b} \cdot a
$$


However, by applying the substitution that maps names $a$ and $b$ to $p$, we obtain processes $p \mid \bar{p}$ and $p \cdot \bar{p}+\bar{p} \cdot p$ respectively, which are not bisimilar: the former can do a $\tau$ transition that cannot be matched by the latter. Actually, $a \cdot \bar{b}+\bar{b} \cdot a$ gives a simple example of a mutual desynchronisation in $\mu \mathrm{CCS}^{+}$. This standard counterexample to substitution closure essentially explains why early and late bisimilarities are not congruences in the (full) $\pi$ calculus.

Remark 4.6 (Restriction and replication instead of choice). As shown in [15, a related counterexample can be constructed if, instead of adding the sum operator, we add restriction and replication to $\mu \mathrm{CCS}$ : the equivalence

$$
! a . \bar{b} . \tau . q \mid ! \bar{b} . a . \tau . q \sim !(\boldsymbol{\nu} c)(a . \bar{c} \mid \bar{b} . c . q)
$$

fails to hold if we replace $a$ and $b$ with $p$, because one process is liable to do two synchronisations and interact on $q$, while the other one needs at least three synchronisations to do so (the construction $\tau . P$ can be encoded as $(\boldsymbol{\nu} d)(d . P \mid \bar{d})$, for some fresh channel name $d$ ).

4.2. Noninterleaving Semantics. We shall work in $\mu \mathrm{CCS}^{+}$in the remainder of this section. It can be remarked that equality (4.1) - which is an instance of the expansion law - is typical of interleaving semantics, in which the parallel composition of two processes is equivalent to a single process, that expresses using nondeterminism all possible interleavings of the two concurrent activities. As we have seen, equivalences that validate (4.1), as is the case for strong bisimilarity in $\mu \mathrm{CCS}^{+}$, are usually not substitution closed.

On the contrary, we can expect locality-aware semantics, that are sensitive to the parallel structure of processes (and hence more discriminating than $\sim$ ), to be closed under substitution. There are several approaches to define such equivalences. We focus here on a version of (strong) distributed bisimilarity [5, 4, because it is among the simplest, and this will suffice for our purposes. The definition of distributed bisimilarity relies on distributed transitions, which are given by judgements of the form $P \stackrel{\mu}{\rightarrow}$ d $\left\langle P_{1}, P_{2}\right\rangle$. The intended meaning is that when $P$ performs the transition along $\mu$, it is decomposed into two parts. At the site where the transition has happened, the local process evolves into $P_{1}$ (the local residual). The remainder of the process, which has not taken part in the transition, evolves into $P_{2}$ (the concurrent residual). For example, we have $P_{1}|\eta \cdot Q| P_{2} \stackrel{\eta}{\rightarrow}_{\mathrm{d}}\left\langle Q, P_{1} \mid P_{2}\right\rangle$.

The inference rules for distributed transitions in $\mu \mathrm{CCS}^{+}$are the following (symmetrical versions of the rules for sum and parallel composition are omitted):

$$
\begin{aligned}
& \eta . P \stackrel{\eta}{\rightarrow}_{\mathrm{d}}\langle P, \mathbf{0}\rangle \quad \frac{S \stackrel{\eta}{\rightarrow}_{\mathrm{d}}\left\langle P_{1}, P_{2}\right\rangle}{S+S^{\prime} \stackrel{\eta}{\rightarrow}_{\mathrm{d}}\left\langle P_{1}, P_{2}\right\rangle} \quad \frac{P \stackrel{\eta}{\rightarrow}_{\mathrm{d}}\left\langle P_{1}, P_{2}\right\rangle}{P \mid P^{\prime} \stackrel{\eta}{\rightarrow}_{\mathrm{d}}\left\langle P_{1}, P_{2} \mid P^{\prime}\right\rangle} \\
& \frac{P \stackrel{\eta}{\rightarrow}{ }_{\mathrm{d}}\left\langle P_{1}, P_{2}\right\rangle \quad Q \stackrel{\stackrel{\bar{\eta}}{\rightarrow}_{\mathrm{d}}}{\rightarrow}\left\langle Q_{1}, Q_{2}\right\rangle}{P \mid Q \stackrel{\stackrel{\tau}{\rightarrow}_{\mathrm{d}}}{{ }_{1}}\left\langle P_{1}\left|Q_{1}, P_{2}\right| Q_{2}\right\rangle}
\end{aligned}
$$

Definition 4.7 (Distributed bisimilarity). A symmetric relation $\mathcal{R}$ between processes is a distributed bisimulation iff whenever $P \mathcal{R} Q$, if $P \stackrel{\mu}{\rightarrow}\left\langle P_{1}, P_{2}\right\rangle$, then there exist $Q_{1}, Q_{2}$ such that $Q \stackrel{\mu}{\rightarrow} \mathrm{d}\left\langle Q_{1}, Q_{2}\right\rangle, P_{1} \mathcal{R} Q_{1}$ and $P_{2} \mathcal{R} Q_{2}$.

Distributed bisimilarity, written $\sim_{\mathrm{d}}$, is the greatest distributed bisimulation.

Lemma 4.8. If $P \stackrel{\eta}{\rightarrow}\left\langle P_{1}, P_{2}\right\rangle$, then $P \equiv\left(\eta . P_{1}+S_{1}\right) \mid P_{2}$ for some $S_{1}$. 
Lemma 4.9. $\sim_{\mathrm{d}}$ is a congruence on $\mu C C S^{+}$.

Proposition 4.10. $\sim_{\mathrm{d}}$ is substitution closed in $\mu C C S^{+}$.

Prop.4.10 is established by following the reasoning we have sketched before Def. 4.1, but things are considerably more easy due to distributed transitions, that insure that concurrent prefixes can be fired.

Actually, $\sim_{\mathrm{d}}$ coincides with structural congruence in $\mu \mathrm{CCS}^{+}\left(\right.$in $\mu \mathrm{CCS}^{+}$, in addition to the equalities that are valid in $\mu \mathrm{CCS}$, $\equiv$ satisfies the laws of an abelian monoid for + , as well as the idempotence law $S+S \equiv S$ ). To show this, we first establish the following separation property, enjoyed by $\sim_{\mathrm{d}}$ in $\mu \mathrm{CCS}^{+}$:

Lemma 4.11 (Separation Property). If $P=\prod_{i \in I} S_{i}, Q=\prod_{j \in J} S_{j}^{\prime}$, and $P \sim_{\mathrm{d}} Q$, then there exists a bijection $f$ from $I$ to $J$ such that $\forall i \in I . S_{i} \sim_{\mathrm{d}} S_{f(i)}^{\prime}$.

Proof. We first observe a general property of distributed transitions: for any $i_{0} \in I$, whenever $S_{i_{0}} \stackrel{\mu}{\rightarrow} P_{0}$, by Def. [4.7, we have $S_{j_{0}}^{\prime} \stackrel{\mu}{\rightarrow} Q_{0}$ for some $j_{0}, Q_{0}$, with $P_{0} \sim_{\mathrm{d}} Q_{0}$ and $\prod_{i \in I, i \neq i_{0}} S_{i} \sim_{\mathrm{d}} \prod_{j \in J, j \neq j_{0}} S_{j}^{\prime}$, where the latter equivalence involves processes that have exactly one parallel component less than $P$ and $Q$ respectively. The symmetrical property also holds for challenges coming from $Q$.

Let us now prove that $I$ and $J$ have the same cardinal. We assume without loss of generality that $I$ has strictly more elements than $J$. We derive a contradiction by repeatedly using the remark above to fire challenges in the parallel components of $P$, until there are no components left in $Q . I$ and $J$ thus have the same cardinal.

In light of this result, we can assume w.l.o.g. that $I$ is the set of indices in $P$ 's and $Q$ 's decompositions, and moreover that $0 \in I$. We thus show:

If $P=\prod_{i \in I} S_{i}, Q=\prod_{j \in I} S_{j}^{\prime}$, and $P \sim_{\mathrm{d}} Q$, then there exists a bijection $f$ from $I$ to $J$ such that $\forall i \in I . S_{i} \sim_{\mathrm{d}} S_{f(i)}^{\prime}$.

To prove this, we reason by induction on the number of parallel components of $P$. The cases where this number is 0 or 1 are immediate. Assume then that $I$ has at least two elements. We distinguish two cases:

First case: all components are equivalent to each other on each side, that is, $\forall i \in I . S_{i} \sim_{\mathrm{d}} S_{0}$, and $\forall j \in I . S_{j}^{\prime} \sim_{\mathrm{d}} S_{0}^{\prime}$. It remains to show that one of the $S_{i} \mathrm{~s}$ is equivalent to one of $S_{j}^{\prime} \mathrm{s}$ : for this, we use the remark above about distributed transitions to fire all components of $P$ but one, which gives us that the remaining component is bisimilar to a component of $Q$.

Second case: if we define $C=\left\{i . S_{i} \sim_{\mathrm{d}} S_{0}\right\}$, we have $\emptyset \nsubseteq C \nsubseteq I$ (since otherwise, we would be in the first case). Define $C^{\prime}=I \backslash C$, and perform a sequence of $\sim_{\mathrm{d}}$-challenges on the side of $P$ in order to fire all components corresponding to $C^{\prime}$ : we are left with $\prod_{i \in C} S_{i} \sim_{\mathrm{d}} \prod_{i \in D} S_{i}^{\prime}$ for some $D \nsubseteq I$.

Since $C \nsubseteq I$, we can apply induction to derive that the $S_{i}$ s are one to one equivalent to the $S_{j}^{\prime}$ s, which yields that all processes in $\left\{S_{i}, i \in C\right\} \cup\left\{S_{j}^{\prime}, j \in D\right\}$ belong to the same equivalence class for $\sim_{\mathrm{d}}$ (and are hence all equivalent to $S_{0}$ ).

Similarly, by firing all components in $C$, we obtain $\prod_{i \in C^{\prime}} S_{i} \sim_{\mathrm{d}} \prod_{i \in D^{\prime}} S_{i}^{\prime}$ for $D^{\prime} \nsubseteq I$. Again, as $C^{\prime} \nsubseteq I$, we have by induction that every element in $\left\{S_{i}, i \in C^{\prime}\right\}$ is in one to one correspondence with an element of $\left\{S_{j}^{\prime}, j \in D^{\prime}\right\}$. This implies, by definition of $C^{\prime}$, that none of the $S_{j}^{\prime}$ s for $j \in D^{\prime}$ is equivalent to $S_{0}$. Hence, we have that $D \cap D^{\prime}=\emptyset$, and $D \cup D^{\prime}=I$ by a cardinality argument; the announced property follows. 
Lemma 4.11 formalises the fact that in absence of restriction, distributed bisimilarity is discriminating enough to analyse the maximum degree of parallelism in processes (in particular, the expansion law is not valid for location sensitive equivalences).

Proposition 4.12. In $\mu C C S^{+}, P \sim_{\mathrm{d}} Q$ if and only if $P \equiv Q$.

Proof. We first remark that $\equiv \subseteq \sim_{\mathrm{d}}$ on $\mu \mathrm{CCS}^{+}$. To show the converse, we assume $P \sim_{\mathrm{d}} Q$, and reason by induction on the size of $P$, defined as the number of prefixes in $P$. The cases where $P$ is of size 0 or 1 are immediate. Assume then that the size of $P$ is strictly greater than 1.

First, if $P$ has at least two parallel components that are different from $\mathbf{0}$, we can apply the separation property (Lemma 4.11), together with the induction hypothesis, to deduce the expected result.

Assume now $P=\sum_{i \in I} \eta_{i} . P_{i}$. By Lemma 4.11, $Q$ has only one parallel component, i.e., $Q=\sum_{j \in J} \eta_{i}^{\prime} \cdot Q_{i}$. Using the idempotence law $(S+S \equiv S)$, we moreover assume w.l.o.g. that for all $i_{1}, i_{2}, \eta_{i_{1}} \cdot P_{i_{1}} \equiv \eta_{i_{2}} . P_{i_{2}}$ implies $i_{1}=i_{2}$, and similarly for the summands of $Q$.

Since $P \sim_{\mathrm{d}} Q$, we observe two properties. First, $\forall i \in I . \exists j \in J . \eta_{i}=\eta_{j}^{\prime} \wedge P_{i} \sim_{\mathrm{d}} Q_{j}$ : this follows by firing a challenge on $\eta_{i}$ on $P$ 's side. Symmetrically, $\forall j \in J$. $\exists i \in I \eta_{i}=\eta_{j}^{\prime} \wedge P_{i} \sim_{\mathrm{d}}$ $Q_{j}$. In each case, the induction hypothesis actually gives $P_{i} \equiv Q_{j}$.

Now, for any $i_{1} \in I$, the first property associates some $j \in J$ to $i$, which in turn is associated to $i_{2} \in I$ by the second property. In this case, we have $\eta_{i_{1}}=\eta_{j}=\eta_{i_{2}}$ and $P_{i_{1}} \equiv Q_{j} \equiv P_{i_{2}}$, which insures $i_{1}=i_{2}$ by the hypothesis we have made. A similar argument, starting from $Q$ 's side, shows that these two properties entail that the summands in $P$ are in one to one correspondence with the summands of $Q$, whence, finally, $P \equiv Q$.

In view of this result, $\sim_{\mathrm{d}}$ is arguably not very interesting in $\mu \mathrm{CCS}^{+}$. The main point here is to show a situation where $\sim$ is not substitution closed, while $\sim_{\mathrm{d}}$ is. It can be proved (but this requires more work) that the same holds if we move to a richer calculus, where parallel compositions are allowed in summands. In such a calculus, $\sim_{\mathrm{d}}$ satisfies nontrivial absorption laws, such as $a . b \mid \bar{a} . c \sim_{\mathrm{d}}(a . b \mid \bar{a} . c)+\tau .(b \mid c)$, which is obviously not valid for $\equiv$ (we suppose here the existence of a $\tau$ prefix; more general absorption laws can be defined see [4]).

One way to establish that $\sim_{\mathrm{d}}$ is closed under substitution in the richer calculus is to exploit the results of [4, Sect. 4.5], which studies axiomatisations of $\sim_{\mathrm{d}}$. These axiomatisations use a new operator, noted $\nmid$, that satisfies the following laws:

$$
\begin{array}{cl}
(P+Q) \nmid R=P \nmid R+Q \nmid R & (P \nmid Q) \nmid R=P \nmid(Q \mid R) \\
P \nmid \mathbf{0}=P & \mathbf{0} \nmid P=\mathbf{0}
\end{array}
$$

$\nmid$ is a kind of asymmetric parallel composition, that intuitively gives precedence to the transitions of its left hand side operand. Moreover, as shown in [4], if we allow communications across $\nmid$, then the following expansion theorem

$$
\begin{aligned}
& \text { If } P=\sum_{i \in I} \eta_{i} \cdot P_{i} \nmid P_{i}^{\prime} \text { and } Q=\sum_{j \in J} \eta_{j}^{\prime} \cdot Q_{j} \nmid Q_{j}^{\prime} \text {, then } \\
& \qquad P \mid Q=\sum_{i \in I} \eta_{i} \cdot P_{i} \nmid\left(P_{i}^{\prime} \mid Q\right)+\sum_{j \in J} \eta_{j}^{\prime} \cdot Q_{j} \nmid\left(P \mid Q_{j}^{\prime}\right)+\sum_{\eta_{i}=\eta_{j}^{\prime}} \tau \cdot\left(P_{i} \mid Q_{j}\right) \nmid\left(P_{i}^{\prime} \mid Q_{j}^{\prime}\right)
\end{aligned}
$$


together with the laws of $\nmid$ and of + , provides a complete axiomatisation of $\sim_{\mathrm{d}}$.

This expansion theorem closely resembles its standard counterpart in interleaving semantics, where concurrency is expressed using the sum operator. However, since communications are allowed between the operands of $\nmid$, the above equality is robust w.r.t. substitution. Indeed, if a new interaction is triggered on the left hand side of the equality by applying a substitution, say between $\eta_{i} \cdot P_{i}$ and $\eta_{j}^{\prime} . Q_{j}$, then this synchronisation is also possible on the right hand side (in the first summand). We do not enter any further into the details of this proof.

\section{A New Congruence Result for the $\pi$-Calculus}

5.1. The Finite, Sum-free $\pi$-calculus. Processes of $\pi_{0}$ are built from an infinite set $\mathcal{N}_{\pi}$ of names (we let $a, b \ldots, m, n \ldots, p, q \ldots, x, y \ldots$ range over names), according to the following grammar:

$$
\phi::=m(x)|\bar{m} n, \quad P::=\mathbf{0}| \phi . P\left|P_{1}\right| P_{2} \mid(\boldsymbol{\nu} p) P .
$$

The input prefix $m(x)$ binds name $x$ in the continuation process, and so does name restriction $(\boldsymbol{\nu} n)$ in the restricted process. A name that is not bound is said to be free, and we let $\operatorname{fn}(P)$ stand for the free names of $P$. We assume that any process that we manipulate satisfies a Barendregt convention: every bound name is distinct from the other bound and free names of the process. We shall use $a, b, c$ to range over free names of processes, $p, q, r$ (resp. $x, y)$ to range over names bound by restriction (resp. by input), and $m, n$ to range over any name, free or bound (note that these naming conventions are used in the above grammar). Structural congruence on $\pi_{0}$, written $\equiv$, is the smallest congruence that is an equivalence relation, contains $\alpha$-equivalence, and satisfies the following laws:

$$
\begin{array}{cccc}
P \mid \mathbf{0} \equiv P & P|(Q \mid R) \equiv(P \mid Q)| R & P|Q \equiv Q| P & (\boldsymbol{\nu} p) \mathbf{0} \equiv \mathbf{0} \\
(\boldsymbol{\nu} p)(\boldsymbol{\nu} q) P \equiv(\boldsymbol{\nu} q)(\boldsymbol{\nu} p) P & P \mid(\boldsymbol{\nu} p) Q \equiv(\boldsymbol{\nu} p)(P \mid Q) & \text { if } p \notin \mathrm{fn}(P)
\end{array}
$$

We let $P[n / x]$ stand for the capture avoiding substitution of name $x$ with name $n$ in $P$. We use $\sigma$ to range over substitutions in $\pi_{0}$ (that simultaneously replace several names).

Definition 5.1 (Late operational semantics and ground bisimilarity). The late operational semantics of $\pi_{0}$ is given by a transition relation whose set of labels is defined by:

$$
\mu::=a(x)|\bar{a} b| \bar{a}(p) \mid \tau \text {. }
$$

Names $x$ and $p$ are said to be bound in actions $a(x)$ and $\bar{a}(p)$ respectively, and other names are free. We use $\operatorname{bn}(\mu)$ (resp. $\mathrm{fn}(\mu))$ to denote the set of bound (resp. free) names of action $\mu$. 
The late transition relation, written $\rightarrow_{\pi}$, is given by the following rules (symmetrical versions of the rules involving parallel composition are omitted):

$$
\begin{aligned}
& \underset{\phi . P \stackrel{\phi}{\rightarrow}_{\pi} P}{P} \frac{P \stackrel{a(x)}{\rightarrow} P^{\prime} \quad Q \stackrel{\bar{a} b}{\rightarrow} Q^{\prime}}{P\left|Q \stackrel{\tau}{\rightarrow}_{\pi} P^{\prime}[b / x]\right| Q^{\prime}} \\
& \frac{P \stackrel{\bar{a} b}{\longrightarrow}_{\pi} P^{\prime}}{(\boldsymbol{\nu} b) P \stackrel{\bar{a}(b)}{\longrightarrow}_{\pi} P^{\prime}} a \neq b \quad \frac{P \stackrel{a(x)}{\longrightarrow}_{\pi} P^{\prime} \quad Q \stackrel{\bar{a}(p)}{\rightarrow}_{\pi} Q^{\prime}}{P \mid Q \stackrel{\tau}{\rightarrow}_{\pi}(\boldsymbol{\nu} p)\left(P^{\prime}[p / x] \mid Q^{\prime}\right)} \\
& \frac{P \stackrel{\mu}{\rightarrow}_{\pi} P^{\prime}}{P\left|Q \stackrel{\mu}{\rightarrow}_{\pi} P^{\prime}\right| Q} \operatorname{bn}(\mu) \cap \mathrm{fn}(Q)=\emptyset \quad \frac{P \stackrel{\mu}{\rightarrow}_{\pi} P^{\prime}}{(\boldsymbol{\nu} p) P \stackrel{\mu}{\rightarrow}_{\pi}(\boldsymbol{\nu} p) P^{\prime}} p \notin \operatorname{fn}(\mu)
\end{aligned}
$$

A ground bisimulation is a symmetric relation $\mathcal{R}$ between processes such that whenever $P \mathcal{R} Q$ and $P \stackrel{\mu}{\rightarrow} \pi P^{\prime}$, there exists $Q^{\prime}$ s.t. $Q \stackrel{\mu}{\rightarrow}_{\pi} Q^{\prime}$ and $P^{\prime} \mathcal{R} Q^{\prime}$.

Ground bisimilarity, written $\sim_{\mathrm{g}}$, is the union of all ground bisimulations.

Note that we do not respect the convention on names in the rule to infer a bound output, precisely because we are transforming a free name (b) into a bound name.

Lemma 5.2. Assume that $P \sigma \stackrel{\mu}{\rightarrow} P^{\prime}$.

(1) If $\mu$ is $\bar{a} b, \bar{a}(p)$ or $a(x)$, then $P{\stackrel{\mu^{\prime}}{\longrightarrow}}_{\pi} P^{\prime \prime}$ with $\mu^{\prime} \sigma=\mu$ and $P^{\prime \prime} \sigma=P^{\prime}$.

(2) If $\mu=\tau$ then one of the three following properties hold, where the input and output actions are offered concurrently by $P$ in the last two cases.

(a) $P \stackrel{\tau}{\rightarrow}_{\pi} P^{\prime \prime}$ and $P^{\prime \prime} \sigma=P^{\prime}$,

(b) $P \stackrel{\bar{b} c}{\longrightarrow}_{\pi} \stackrel{a(x)}{\longrightarrow}_{\pi} P^{\prime \prime}$ where $\sigma(a)=\sigma(b)$ and $P^{\prime \prime}[c / x] \sigma \sim P^{\prime}$,

(c) $P \stackrel{\bar{b}(p)}{\longrightarrow}_{\pi} \stackrel{a(x)}{\longrightarrow}_{\pi} P^{\prime \prime}$ where $\sigma(a)=\sigma(b)$ and $\left((\boldsymbol{\nu} p) P^{\prime \prime}[p / x]\right) \sigma \sim P^{\prime}$.

Proof. Similar to the proof of Lemma 1.4.13 in [15], where the early transition semantics is treated.

5.2. Mutual Desynchronisations in $\pi_{0}$. In what follows, we fix two distinct names $a$ and $b$, that will occur free in the processes we shall consider. The definitions and results below will depend on $a$ and $b$, but we avoid making this dependency explicit, in order to ease readability. Names $a$ and $b$ will be fixed in the proof of Theorem 5.8.

Definition 5.3 (Erasing a $\pi_{0}$ process). Given a $\pi_{0}$ process $P$, we define the erasing of $P$, written $\mathcal{E}(P)$, as follows:

$$
\begin{aligned}
& \mathcal{E}\left(P_{1} \mid P_{2}\right) \stackrel{\text { def }}{=} \mathcal{E}\left(P_{1}\right) \mid \mathcal{E}\left(P_{2}\right) \quad \mathcal{E}((\boldsymbol{\nu} p) P) \stackrel{\text { def }}{=} \mathcal{E}(P) \quad \mathcal{E}(\mathbf{0}) \stackrel{\text { def }}{=} \mathbf{0} \\
& \mathcal{E}(a(x) . P) \stackrel{\text { def }}{=} a \cdot \mathcal{E}(P) \quad \mathcal{E}(m(x) . P) \stackrel{\text { def }}{=} \mathbf{0} \text { if } m \neq a \\
& \mathcal{E}(\bar{b} n . P) \stackrel{\text { def }}{=} \bar{b} \cdot \mathcal{E}(P) \quad \mathcal{E}(\bar{m} n . P) \stackrel{\text { def }}{=} \mathbf{0} \text { if } m \neq b
\end{aligned}
$$


Note that $a$ and $b$ play different roles in the definition of $\mathcal{E}(\cdot)$.

It is immediate from the definition that $\mathcal{E}(P)$ is a $\mu \mathrm{CCS}$ process whose only prefixes are $a$ and $\bar{b}$. Intuitively, $\mathcal{E}(P)$ only exhibits the interactions of $P$ at $a$ (in input) and $b$ (in output) that are not guarded by interactions on other names.

Lemma 5.4 (Transitions of $\mathcal{E}(P)$ ). Consider a $\pi_{0}$ process $P$. We have:

- If $P \stackrel{a(x)}{\longrightarrow} P^{\prime}$, then $\mathcal{E}(P) \stackrel{a}{\rightarrow} \mathcal{E}\left(P^{\prime}\right)$.

- If $P \stackrel{\bar{b} c}{\longrightarrow}_{\pi} P^{\prime}$ or $P \stackrel{\bar{b}(p)}{\longrightarrow}_{\pi} P^{\prime}$, then $\mathcal{E}(P) \stackrel{\bar{b}}{\rightarrow} \mathcal{E}\left(P^{\prime}\right)$.

- Conversely, if $\mathcal{E}(P) \stackrel{a}{\rightarrow} P_{0}$, then there exist $x$ and $P^{\prime}$ such that $P_{0}=\mathcal{E}\left(P^{\prime}\right)$ and $P \stackrel{a(x)}{\longrightarrow} P_{\pi}$. Similarly, if $\mathcal{E}(P) \stackrel{\bar{b}}{\rightarrow} P_{0}$, there exist $c, p, P^{\prime}$ such that $P_{0}=\mathcal{E}\left(P^{\prime}\right)$ and either $P \stackrel{\bar{b} c}{\longrightarrow}{ }_{\pi} P^{\prime}$ or $P \stackrel{\bar{b}}{(p)}_{\pi} P^{\prime}$.

Proof. Simple reasoning on the LTSs of $\mu \mathrm{CCS}$ and $\pi_{0}$.

Proposition 5.5 (Transfer). If $P \sim_{\mathrm{g}} Q$ in $\pi_{0}$, then $\mathcal{E}(P) \sim \mathcal{E}(Q)$ in $\mu C C S$.

Proof. We reason by induction on the size of $P$ (defined as the number of prefixes in $P$ ). Consider a transition of $\mathcal{E}(P)$; as observed above, it can only be a transition along $a$ or a transition along $\bar{b}$.

Assume $\mathcal{E}(P) \stackrel{a}{\rightarrow} P_{0}$. By Lemma [5.4, $P \stackrel{a(x)}{\longrightarrow}{ }_{\pi} P^{\prime}$ and $P_{0}=\mathcal{E}\left(P^{\prime}\right)$. Since $P \sim_{\mathrm{g}} Q$, $Q \stackrel{a(x)}{\longrightarrow} Q^{\prime}$ for some $Q^{\prime}$ such that $P^{\prime} \sim_{\mathrm{g}} Q^{\prime}$. By induction, the latter relation gives $\mathcal{E}\left(P^{\prime}\right) \sim \mathcal{E}\left(Q^{\prime}\right)$, and $Q \stackrel{a(x)}{\longrightarrow}{ }_{\pi} Q^{\prime}$ gives by Lemma $5.4 \mathcal{E}(Q) \stackrel{a}{\rightarrow} \mathcal{E}\left(Q^{\prime}\right)$.

The case $\mathcal{E}(P) \stackrel{\bar{b}}{\rightarrow} P_{0}$ is treated similarly: by Lemma 5.4, there are two cases, according to whether $P$ does a free output or a bound output. Reasoning like above allows us to conclude in both cases.

We can now present our central technical result about $\pi_{0}$, which comes in two lemmas.

Lemma 5.6. If $Q \sim_{\mathrm{g}}(\boldsymbol{\nu} \widetilde{p})\left(a(x) . P_{1}\left|\bar{b} c . P_{2}\right| P_{3}\right)$, then there exist some $Q_{1}, Q_{2}, Q_{3}, \widetilde{q}$, such that $Q \equiv(\boldsymbol{\nu} \widetilde{q})\left(a(x) \cdot Q_{1}\left|\bar{b} c \cdot Q_{2}\right| Q_{3}\right)$ and

$$
(\boldsymbol{\nu} \widetilde{p})\left(P_{1}\left|P_{2}\right| P_{3}\right) \sim_{\mathrm{g}}(\boldsymbol{\nu} \widetilde{q})\left(Q_{1}\left|Q_{2}\right| Q_{3}\right) .
$$

Proof. Let $P=(\boldsymbol{\nu} \widetilde{p})\left(a(x) \cdot P_{1}\left|\bar{b} c . P_{2}\right| P_{3}\right)$ and $P^{\prime}=(\boldsymbol{\nu} \widetilde{p})\left(P_{1}\left|P_{2}\right| P_{3}\right)$.

Note that by our conventions on notations, $c \notin \widetilde{p}$.

Since $Q \sim_{\mathrm{g}} P$ and $P$ can perform two transitions along $a(x)$ and $\bar{b} c$ respectively, $Q$ can also perform these transitions, which gives

$Q \equiv(\boldsymbol{\nu} \widetilde{q})\left(a(x) \cdot Q_{1}\left|\bar{b} c \cdot Q_{2}\right| Q_{3}\right)$ for some $\widetilde{q}, Q_{1}, Q_{2}, Q_{3}$,

the first (resp. second) component exhibiting the prefix that is triggered to answer the challenge on $a(x)$ (resp. $\bar{b}$ ).

Consider now the challenge $P \stackrel{\bar{b} c}{\longrightarrow} \stackrel{a(x)}{\longrightarrow}_{\pi} P^{\prime}$, to which $Q$ answers by performing the transition $Q \stackrel{\bar{b} c}{\longrightarrow} \stackrel{a(x)}{\longrightarrow}_{\pi} Q_{b a}$, with $P^{\prime} \sim_{\mathrm{g}} Q_{b a}$. If $Q_{b a}=(\boldsymbol{\nu} \widetilde{q})\left(Q_{1}\left|Q_{2}\right| Q_{3}\right)$, that is, if $Q$ triggers the prefixes on top of its first and second components, then we are done. Similarly, if $Q$ triggers a prefix in $Q_{3}$ to answer the second challenge, say $Q_{3}=a(x) \cdot Q_{4} \mid Q_{5}$, we can set $Q_{1}^{\prime}=a(x) \cdot Q_{4}$ and $Q_{3}^{\prime}=Q_{1} \mid Q_{5}$, and the lemma is proved. 
The case that remains to be analysed is when $Q_{2} \stackrel{a(x)}{\longrightarrow} Q_{2}^{\prime}$ and we have $Q_{b a}=$ $(\boldsymbol{\nu} \widetilde{q})\left(a(x) \cdot Q_{1}\left|Q_{2}^{\prime}\right| Q_{3}\right) \sim_{\mathrm{g}}(\boldsymbol{\nu} \widetilde{p})\left(P_{1}\left|P_{2}\right| P_{3}\right)$.

We then consider the challenge where $P$ fires its two topmost prefixes $a(x)$ and $\bar{b} c$ in the other sequence, namely $P \stackrel{a(x)}{\longrightarrow} \stackrel{\bar{b}}{c}_{\pi} P^{\prime}$. By hypothesis, $Q$ triggers the prefix of its first component for the first transition. To perform the second transition, $Q$ can fire the prefix $\bar{b} c$ either in its second or third component, in which case, as above, we are done, or, and this is the last possibility, the prefix $\bar{b} c$ occurs in $Q_{1}$. This means $Q_{a b}=(\boldsymbol{\nu} \widetilde{q})\left(Q_{1}^{\prime}\left|\bar{b} c \cdot Q_{2}\right| Q_{3}\right) \sim_{\mathrm{g}}$ $(\boldsymbol{\nu} \widetilde{p})\left(P_{1}\left|P_{2}\right| P_{3}\right)$, with $Q_{1} \stackrel{\bar{b} c}{\longrightarrow}_{\pi} Q_{1}^{\prime}$.

To sum up, we have $Q_{a b}=(\boldsymbol{\nu} \widetilde{q})\left(Q_{1}^{\prime}\left|\bar{b} c \cdot Q_{2}\right| Q_{3}\right) \sim_{\mathrm{g}}(\boldsymbol{\nu} \widetilde{q})\left(a(x) \cdot Q_{1}\left|Q_{2}^{\prime}\right| Q_{3}\right)=Q_{b a}$, with $Q_{1} \stackrel{\bar{b} c}{\longrightarrow} Q_{1}^{\prime}$ and $Q_{2} \stackrel{a(x)}{\longrightarrow} Q_{2}^{\prime}$ : this resembles the mutual desynchronisation of Definition 4.1 , translated into the $\pi$-calculus.

Indeed, we can construct a mutual desynchronisation in $\mu$ CCS: $Q_{a b} \sim_{\mathrm{g}} Q_{b a}$ implies $\mathcal{E}\left(Q_{a b}\right) \sim \mathcal{E}\left(Q_{b a}\right)$ by Prop. 5.5, and $Q_{1} \stackrel{\bar{b} c}{\longrightarrow}_{\pi} Q_{1}^{\prime}$ (resp. $Q_{2} \stackrel{a(x)}{\longrightarrow} Q_{2}^{\prime}$ ) implies by Lemma 5.4 $\mathcal{E}\left(Q_{1}\right) \stackrel{\bar{b}}{\rightarrow} \mathcal{E}\left(Q_{1}^{\prime}\right)$ (resp. $\left.\mathcal{E}\left(Q_{2}\right) \stackrel{a}{\rightarrow} \mathcal{E}\left(Q_{2}^{\prime}\right)\right)$. Finally, using Lemma 4.4, we obtain a contradiction, which concludes our proof.

Lemma 5.7. If $Q \sim_{\mathrm{g}}(\boldsymbol{\nu} p, \widetilde{p})\left(a(x) . P_{1}\left|\bar{b} p . P_{2}\right| P_{3}\right)$, then there exist some $Q_{1}, Q_{2}, Q_{3}$, such that $Q \equiv(\boldsymbol{\nu} p, \widetilde{q})\left(a(x) \cdot Q_{1}\left|\bar{b} p \cdot Q_{2}\right| Q_{3}\right)$ and

$$
(\boldsymbol{\nu} \widetilde{p})\left(P_{1}\left|P_{2}\right| P_{3}\right) \sim_{\mathrm{g}}(\boldsymbol{\nu} \widetilde{q})\left(Q_{1}\left|Q_{2}\right| Q_{3}\right) .
$$

Hint. The proof follows the same lines as for the previous lemma. The only difference is when analysing the transitions that lead to $Q_{a b}$ : to perform the second transition, $Q$ can either extrude the name called $p$ in the equality $Q \equiv(\boldsymbol{\nu} p, \widetilde{q})\left(a(x) \cdot Q_{1}\left|\bar{b} p \cdot Q_{2}\right| Q_{3}\right)$, or otherwise $Q$ can be $\alpha$-converted in order to extrude another name. In the case where $Q$ chooses to extrude a different name, we can assume without loss of generality that the necessary $\alpha$-conversion is a swapping between name $p$ and a name $q_{1} \in \widetilde{q}$, which brings us back to the case where name $p$ is the one being extruded.

The presence of a bound output introduces some notational complications when expressing $Q_{a b}$, but basically it does not affect the proof w.r.t. the proof of Lemma 5.6, because the function $\mathcal{E}(\cdot)$ is not sensitive to name permutations that do not involve $a$ or $b$.

\subsection{Congruence.}

Theorem 5.8 (Closure of $\sim_{\mathrm{g}}$ under substitution). If $P \sim_{\mathrm{g}} Q$ then for any substitution $\sigma$, $P \sigma \sim \mathrm{g} Q \sigma$.

Proof. We prove that the relation $\mathcal{R} \stackrel{\text { def }}{=}\left\{(P \sigma, Q \sigma) \mid P \sim_{\mathrm{g}} Q\right\}$ is a ground bisimulation. We consider $P, Q$ such that $P \sim_{\mathrm{g}} Q$ and assume $P \sigma \stackrel{\mu}{\rightarrow}_{\pi} P_{0}$. We examine the transitions of $P$ that make it possible for $P \sigma$ to do a $\mu$-transition to $P_{0}$.

According to Lemma 5.2, there are two possibilities. The first possibility corresponds to the situation where $\mu$ comes from an action that $P$ can perform, i.e., $P{\stackrel{\mu^{\prime}}{\longrightarrow}}_{\pi} P^{\prime}$ for some $\mu^{\prime}$, with $P^{\prime} \sigma=P_{0}$ and $\mu^{\prime} \sigma=\mu$ (cases 1 and $2 \mathrm{a}$ in Lemma 5.2). Since $P \sim_{\mathrm{g}} Q, Q \stackrel{\mu^{\prime}}{\longrightarrow} Q^{\prime}$ 
and $P^{\prime} \sim_{\mathrm{g}} Q^{\prime}$ for some $Q^{\prime}$. We can prove that $Q \sigma \stackrel{\mu}{\rightarrow} Q^{\prime} \sigma$, and since $P^{\prime} \sim_{\mathrm{g}} Q^{\prime}$ we have $\left(P^{\prime} \sigma, Q^{\prime} \sigma\right) \in \mathcal{R}$.

The second possibility (which corresponds to the difficult case) is given by $\mu=\tau$, where the synchronisation in $P^{\prime}$ has been made possible by the application of $\sigma$. There are in turn two cases, corresponding to whether the synchronisation involves a free or a bound name. In the former case, $P \stackrel{a(x)}{\longrightarrow} \pi P^{\prime}$ and $P \stackrel{\bar{b} c}{\longrightarrow}_{\pi} P^{\prime \prime}$ for some $a, x, b, c, P^{\prime}, P^{\prime \prime}$. This entails $P \equiv(\boldsymbol{\nu} \widetilde{p})\left(a(x) \cdot P_{1}\left|\bar{b} c \cdot P_{2}\right| P_{3}\right)$ for some $\widetilde{p}, P_{1}, P_{2}, P_{3}$, and, since $P \sim_{\mathrm{g}} Q$, we conclude by Lemma 5.6 that $Q \equiv(\boldsymbol{\nu} \widetilde{q})\left(a(x) \cdot Q_{1}\left|\bar{b} c \cdot Q_{2}\right| Q_{3}\right)$ and

$$
(\boldsymbol{\nu} \widetilde{p})\left(P_{1}\left|P_{2}\right| P_{3}\right) \sim_{\mathrm{g}}(\boldsymbol{\nu} \widetilde{q})\left(Q_{1}\left|Q_{2}\right| Q_{3}\right) .
$$

By definition of $\mathcal{R}$, this equivalence implies that we can apply any substitution to these two processes to yield processes related by $\mathcal{R}$, and in particular $[c / x] \sigma$, which gives:

$$
\left((\boldsymbol{\nu} \widetilde{p})\left(P_{1}\left|P_{2}\right| P_{3}\right)\right)[c / x] \sigma \quad \mathcal{R} \quad\left((\boldsymbol{\nu} \widetilde{q})\left(Q_{1}\left|Q_{2}\right| Q_{3}\right)\right)[c / x] \sigma .
$$

Using the Barendregt convention hypothesis, this amounts to

$$
P_{0} \equiv\left((\boldsymbol{\nu} \widetilde{p})\left(P_{1}[c / x]\left|P_{2}\right| P_{3}\right)\right) \sigma \quad \mathcal{R} \quad\left((\boldsymbol{\nu} \widetilde{q})\left(Q_{1}[c / x]\left|Q_{2}\right| Q_{3}\right)\right) \sigma \stackrel{\text { def }}{=} Q_{0}
$$

We can then conclude by checking that $Q \sigma \stackrel{\tau}{\rightarrow}_{\pi} Q_{0}$.

We reason similarly for the case where the synchronisation involves the transmission of a bound name, using Lemma 5.7 instead of Lemma 5.6. We remark that Lemma 5.7 gives $(\boldsymbol{\nu} \widetilde{p})\left(P_{1}\left|P_{2}\right| P_{3}\right) \sim_{\mathrm{g}}(\boldsymbol{\nu} \widetilde{q})\left(Q_{1}\left|Q_{2}\right| Q_{3}\right)$, and in this case $P \sigma \stackrel{\tau}{\rightarrow} \pi(\boldsymbol{\nu} p, \widetilde{p})\left(P_{1}[p / x]\left|P_{2}\right| P_{3}\right) \sigma$ (resp. $\left.Q \sigma \stackrel{\tau}{\rightarrow}_{\pi}(\boldsymbol{\nu} p, \widetilde{q})\left(Q_{1}[p / x]\left|Q_{2}\right| Q_{3}\right) \sigma\right)$. In order to be able to add the restriction on $p$ to the terms given by Lemma 5.7, we rely on the fact that $\sim_{\mathrm{g}}$ is preserved by restriction: $P \sim_{\mathrm{g}} Q$ implies $(\boldsymbol{\nu} p) P \sim_{\mathrm{g}}(\boldsymbol{\nu} p) Q$ for any $P, Q, p$. We can then reason as above to conclude.

Corollary 5.9 (Congruence of bisimilarity in $\pi_{0}$ ). In $\pi_{0}$, ground, early and late bisimilarity coincide and are congruences.

Proof. By a standard argument (see [15]): since $\sim_{\mathrm{g}}$ is closed under substitution, $\sim_{\mathrm{g}}$ is an open bisimulation.

It is known (see [15]) that adding either replication or sum to $\pi_{0}$ yields a calculus where strong bisimilarity fails to be a congruence.

\section{Conclusion}

We have presented an axiomatisation of strong bisimilarity on a small subcalculus of CCS, and a new congruence result for the $\pi$-calculus.

Technically, the notion of mutual desynchronisation is related to substitution closure of strong bisimilarity, as soon as substitutions can create new interactions by identifying two names.

We have shown in Sect. 5 that there exists no mutual desynchronisation in $\pi_{0}$, and that $\sim_{\mathrm{g}}$ is a congruence. It appears that in finite calculi, mutual desynchronisations give rise to counterexamples to substitution closure of strong bisimilarity (cf. Sect. 4.1). The situation is less clear when infinite behaviours can be expressed. For instance, in the extension of $\mu \mathrm{CCS}$ with replication, the process $P \stackrel{\text { def }}{=} ! a \mid ! \bar{b}$ is bisimilar to process $Q \stackrel{\text { def }}{=} ! a \cdot \bar{b} \mid ! \bar{b} \cdot a$, 
which leads to a mutual desynchronisation: we have $Q \stackrel{a}{\rightarrow} \stackrel{\bar{b}}{\rightarrow} \equiv Q \stackrel{\bar{b}}{\rightarrow} \stackrel{a}{\rightarrow} \equiv Q$. This mutual desynchronisation is however 'benign': by firing concurrently the two prefixes that iniate the mutual desynchronisation, we obtain $a|\bar{b}| P$ which is bisimilar to $P$, so that this situation is not problematic w.r.t. substitution closure (we may moreover remark that the two aforementioned processes remain bisimilar when $b$ is replaced with $a$ ). We do not know at present whether $\sim$ is substitution-closed in this extension of $\mu$ CCS.

Some subcalculi of the $\pi$-calculus where strong bisimilarity is a congruence are obtained by restricting the output prefix [15]. In the asynchronous $\pi$-calculus $(A \pi)$, mutual desynchronisations do not appear, basically because the output action is not a prefix. Strong bisimilarity is a congruence on $A \pi$. In the private $\pi$-calculus $(P \pi)$, since only private names are emitted, no substitution generated by a synchronisation can identify two previously distinct names. Hence, although mutual desynchronisations exist in $P \pi$ (due to the presence of the sum operator), strong bisimilarity is not substitution closed, but is a congruence. Indeed, to obtain the latter property, we only need to consider the particular substitutions at work in $P \pi$, which cannot identify two names.

Regarding future extensions of this work, we would like to study whether our approach can be adapted to analyse weak bisimilarity in $\pi_{0}$ (as mentioned in Remark 2.8, strong and weak bisimilarity coincide in $\mu \mathrm{CCS}$ ). Another interesting direction, as hinted above, would be to study strong bisimilarity on infinite, restriction-free calculi (in CCS and the $\pi$-calculus).

Acknowledgements. We are grateful to Arnaud Carayol for interesting discussions at early stages of this work, and to Ilaria Castellani for discussions about distributed bisimilarity. An anonymous FOSSACS referee provided numerous suggestions, which helped us in particular to improve the proof of Theorem 2.6. We benefited from support by the french initiative "ACI GEOCAL" and from the ANR projects "MoDyFiable" and "CHoCO".

\section{REFERENCES}

[1] L. Aceto, W.J. Fokkink, A. Ingolfsdottir, and B. Luttik. Finite Equational Bases in Process Algebra: Results and Open Questions. In Processes, Terms and Cycles: Steps on the Road to Infinity, volume 3838 of LNCS. Springer Verlag, 2005.

[2] M. Boreale and D. Sangiorgi. Some Congruence Properties for $\pi$-calculus Bisimilarities. TCS, 198:159176, 1998.

[3] O. Burkart, D. Caucal, F. Moller, and B. Steffen. Verification over Infinite States. In Handbook of Process Algebra, pages 545-623. Elsevier, 2001.

[4] I. Castellani. Bisimulations for Concurrency. PhD thesis, Edinburgh University, 1988. Available as report CST-51-88 and LFCS-88-51.

[5] I. Castellani and M. Hennessy. Distributed bisimulations. J. ACM, 36(4):887-911, 1989.

[6] F. Corradini, R. Gorrieri, and D. Marchignoli. Towards parallelization of concurrent systems. Informatique Théorique et Applications, 32(4-6):99-125, 1998.

[7] W. Fokkink and B. Luttik. An $\omega$-complete Equational Specification of Interleaving. In Proc. of ICALP'00, volume 1853 of LNCS, pages 729-743. Springer Verlag, 2000.

[8] J. F. Groote. A New Strategy for Proving $\omega$-Completeness Applied to Process Algebra. In Proc. of CONCUR'90, LNCS, pages 314-331. Springer Verlag, 1990.

[9] D. Hirschkoff and D. Pous. A Distribution Law for CCS and a New Congruence Result for the PiCalculus. In Proc. of FoSSaCS'0\%, LNCS, pages 228-242. Springer Verlag, 2007. 
[10] Y. Hirshfeld and M. Jerrum. Bisimulation Equivalence is Decidable for Normed Process Algebra. Technical Report ECS-LFCS-98-386, LFCS, 1998. an abstract has appeared in the proceedings of ICALP'99, LNCS 1644, Springer Verlag.

[11] B. Luttik. What is Algebraic in Process Theory? Concurrency Column, Bulletin of the EATCS, 88, 2006.

[12] R. Milner and F. Moller. Unique Decomposition of Processes. TCS, 107(2):357-363, 1993.

[13] F. Moller. Axioms for Concurrency. PhD thesis, University of Edinburgh, 1988.

[14] D. Sangiorgi. A Theory of Bisimulation for the $\pi$-Calculus. Acta Informatica, 33(1):69-97, 1996.

[15] D. Sangiorgi and D. Walker. The $\pi$-calculus: a Theory of Mobile Processes. Cambridge University Press, 2001.

[16] B. Victor, F. Moller, M. Dam, and L.-H. Eriksson. The Mobility Workbench. available from http://www.it.uu.se/research/group/mobility/mwb, 2006. 